The AstrophysiCAL Journal, 506:533-539, 1998 October 20

(c) 1998. The American Astronomical Society. All rights reserved. Printed in U.S.A.

\title{
DISTINGUISHING BETWEEN BINARY-SOURCE AND PLANETARY MICROLENSING PERTURBATIONS
}

\author{
B. SCOTT GAUDI \\ Department of Astronomy, Ohio State University, Columbus, OH 43210; gaudi@payne.mps.ohio-state.edu \\ Received 1997 June 6; accepted 1998 May 2
}

\begin{abstract}
A planetary microlensing event is characterized by a short-lived perturbation to the standard microlensing curve. Planetary perturbations typically last from a few hours to a day, have maximum amplitudes, $\delta_{\max }$, of $\pm 5 \%-20 \%$ of the standard curve, and come in two classes: major and minor image perturbations. There exist a subset of binary-source events that can reproduce the main features of major image perturbations, which are likely to represent more than half of all planetary events, and thus masquerade as planetary events. These events require a binary source with a small flux ratio, $\epsilon \sim 10^{-2}$ to $10^{-4}$, and a small impact parameter for the fainter source, $\beta_{2} \lesssim \epsilon / \delta_{\max }$. The detection probability of events of this type is $\sim \beta_{2}$, and can be as high as $\sim 30 \%$; this is comparable to planetary detection rates. Thus a sample of planetary-like perturbations could be seriously contaminated by binary-source events, and there exists the possibility that completely meaningless physical parameters would be derived for any given major image perturbation. Here I derive analytic expressions for a binary-source event in the extreme flux-ratio limit and use these to demonstrate the basic degeneracy between binary-source and planet perturbations. I describe how the degeneracy can be broken by dense and accurate sampling of the perturbation, optical/infrared photometry, or spectroscopic measurements.
\end{abstract}

Subject headings: gravitational lensing — planetary systems

\section{INTRODUCTION}

To date more than 100 microlensing events have been detected toward the Galactic bulge by four groups, MACHO (Alcock et al. 1997a), OGLE (Udalski et al. 1997), DUO (Alard 1996), and EROS (Ansari et al. 1996). Some of these events have been detected in real time; both MACHO and OGLE issue "alerts," notification of ongoing events that have been detected before the peak. These alerts have enabled two follow-up groups, PLANET (Albrow et al. 1996; 1998) and GMAN (Alcock et al. 1997b), to organize worldwide networks devoted to making densely sampled observations of ongoing events. One of the main goals of these groups is to discover planets by searching for shortduration, often small, perturbations on the light curves of alerted events. These perturbations are the signatures of planetary events. While standard microlensing events last from one week to a few months, planetary perturbations are only expected to last a day or less. Thus the need for the intensive, nearly round-the-clock monitoring.

Previous work on planetary microlensing has focused on characterization of the light curves of planetary perturbations (Wambsganss 1997), the criteria for detection of these perturbations (Mao \& Paczyński 1991; Gould \& Loeb 1992; Bolatto \& Falco 1994; Bennett \& Rhie 1996), and the number of systems one might hope to detect based on these criteria (Peale 1997). Unfortunately, mere detection of a perturbation is not sufficient; to have any confidence that a planet has actually been detected, one must determine with reasonable accuracy the physical parameters of the planetary system that can be derived from the event, the planet/star mass ratio $q$, and the planet/star projected separation in units of the Einstein ring $y$. Dominik (1997) discusses ambiguities in the fits of binary lenses, of which planetary systems are a subset. Gaudi \& Gould (1997b) demonstrated that there exist several degeneracies which hamper the determination of $q$ and $y$, including a severe degeneracy that can result in an uncertainty in the derived mass ratio of a factor of $\sim 20$.

Here I discuss an additional degeneracy: a special subset of binary-source events can produce light curves that closely resemble those produced by more than half of planet/star lens systems. This subset, which I will call extreme flux-ratio binary-source events, can produce standard light curves with small, short-duration, positive perturbations. These perturbations can reproduce the gross features of one class of planetary perturbations: major image perturbations. As I discuss in $\S 5.1$, the other class of planetary perturbations, minor image perturbations cannot be reproduced by binary sources. However, minor image perturbations are likely to represent considerably less than half of all detected planetary perturbations. For a binary-source event to mimic a planetary event, the sources must have a small flux ratio, $\epsilon$, and the fainter source must pass close to the lens, with an impact parameter $\beta_{2} \lesssim \epsilon / \delta_{\max }$, where $\delta_{\max }$ is the maximum fractional deviation from the unperturbed light curve. The detection probability for these events is $\sim \beta_{2}$. For $\epsilon \sim 0.01$ and $\delta_{\max } \sim 0.05$, the probability is $\sim 20 \%$. This is comparable to the detection probability of Jupiter-mass planets (Gould \& Loeb 1992). As I discuss in $\S 6,40 \%$ of all sources may be binaries with flux ratios consistent with those required to mimic planetary perturbations. Thus binary stars could seriously contaminate a sample of suspected planetary events. Furthermore, for any given perturbation, there exists the possibility that one could derive completely meaningless physical parameters, since one would not know a priori whether the perturbation was due to a binary source or a planet. For these reasons, it is essential to break this degeneracy and determine the true cause of the perturbation (binary source or planet).

In $\S 2$ I derive analytic expressions for the perturbation due to a binary source in the extreme flux-ratio limit. I use these expressions in $\S 3$ to illustrate the basic degeneracy. In $\S 4$ I estimate the detection probability for extreme fluxratio binary-source events, in $\S 5 \mathrm{I}$ describe methods of 
breaking the degeneracy, and in $\S 6$ I describe how a binarysource event can be used to extract additional information about the lens.

\section{BINARY-SOURCE MICROLENSING IN THE EXTREME FLUX-RATIO LIMIT}

\subsection{Basic Formalism}

The basic formalism for binary-source events has been described in detail by Griest \& Hu (1992) for static binaries and by Han \& Gould (1997) for rotating binaries. Here I briefly review the general formalism and use this formalism to derive the equations for the extreme flux-ratio limit.

The flux of a point source being microlensed by a point mass is given by $F=A F_{0}$, where $F_{0}$ is the umagnified flux and $A$ is the magnification. (Here I ignore any contribution from unresolved sources.) The magnification is a function of the distance of the lens from the observer-source line of sight projected on the lens plane, $u$, which is in turn a function of time:

$$
A[u(t)]=\frac{u^{2}+2}{u\left(u^{2}+4\right)^{1 / 2}} \rightarrow \frac{1}{u}, \quad u(t)^{2}=\left[\frac{\left(t-t_{0}\right)}{t_{\mathrm{E}}}\right]^{2}+\beta^{2} .
$$

The limit applies when $u \ll 1$. Here the impact parameter, $\beta$, and $u$ are in units of the Einstein ring,

$$
r_{\mathrm{E}}^{2}=\frac{4 G M}{c^{2}} \frac{D_{\mathrm{ol}} D_{\mathrm{ls}}}{D_{\mathrm{os}}},
$$

where $M$ is the mass of the lens, and $D_{\mathrm{ol}}, D_{\mathrm{ls}}$, and $D_{\mathrm{os}}$ are the distances between the observer, lens, and source. The characteristic timescale is $t_{\mathrm{E}}=r_{\mathrm{E}} / v$, where $v$ is the transverse velocity of the lens relative to the observer-source line of sight.

For a binary source, the resulting light curve is simply a superposition of two standard light curves, $F=A_{1} F_{0,1}$ $+A_{2} F_{0,2}$ (Griest \& Hu 1992). Henceforth I will assume that $F_{0,2}<F_{0,1}$ and refer to sources 1 and 2 as the primary and secondary, respectively. I define $\epsilon \equiv F_{0,2} / F_{0,1}$. The total magnification is thus

$$
A_{\mathrm{tot}}=\frac{A_{1}+\epsilon A_{2}}{1+\epsilon} .
$$

I define $b$ to be the separation of the sources projected onto the lens plane in units of $r_{\mathrm{E}}$, and $\theta$ to be the angle between the path of the primary and the binary-source axis. Assuming the binary is static, the position of the primary is given by equation (2.1), and the position of the secondary is

$$
u_{2}^{2}=\left[\frac{\left(t-t_{0}\right)}{t_{\mathrm{E}}}+b \cos \theta\right]^{2}+\left(\beta_{1}+b \sin \theta\right)^{2},
$$

where $t_{0}$ is the time of maximum magnification of the primary, and $\beta_{1}$ is the impact parameter of the primary. Without loss of generality, I will assume that $t_{0}=0$.

I now concentrate on cases such that $\epsilon \ll 1$, i.e., where the magnification of the secondary produces a small perturbation to the primary light curve. The fractional deviation of such a binary-source event from the best-fit single-source curve is defined to be $\delta=\left(A_{\mathrm{tot}}-A_{\mathrm{bf}}\right) / A_{\mathrm{bf}}$, where $A_{\mathrm{bf}}$ is the best-fit curve. For $\epsilon \ll 1$, equation (2.3) implies that $\delta \simeq$ $\epsilon A_{2} / A_{1}$. For $\delta$ to be significant, $A_{2} \gg A_{1}$, and the secondary must therefore pass very close to the lens, i.e., $\left|\beta_{2}\right|=$ $\left|\beta_{1}+b \sin \theta\right| \ll 1$. In this limit, equation (2.1) implies that
$A_{2} \sim 1 / u_{2}$, and thus when $\delta$ is significant, $\delta \simeq \epsilon / u_{2} A_{1}^{-1}$. The maximum fractional deviation, $\delta_{\max } \simeq \epsilon / \beta_{2} A_{1}$, occurs when $u_{2}=\beta_{2}$, at time $t_{\max }=-b(\cos \theta) t_{\mathrm{E}}$. The half-maximum occurs when $\delta=\delta_{\max } / 2$, or $u_{2}=2 \beta_{2} A_{1}\left(\beta_{2}\right) / A_{1}\left(u_{2}\right)$. For perturbations with short durations, the magnification of the primary changes only very slowly during the course of the peturbation. Thus $A_{1}$ is roughly the same at $\delta_{\max }$ and at $\delta_{\text {max }} / 2: A_{1}\left(\beta_{2}\right) \sim A_{1}\left(u_{2}\right)$. Thus $u_{2}=2 \beta_{2}$, and the full-width half-maximum (FWHM) of the perturbation is $\tau_{\text {eff }} \simeq$ $12^{1 / 2} \beta_{2} t_{\mathrm{E}}$. The equations governing binary sources in the extreme flux limit are

$$
\begin{gathered}
\delta=\frac{\epsilon}{u_{2}} \frac{1}{A_{1}}, \quad \delta_{\max }=\frac{\epsilon}{\beta_{2}} \frac{1}{A_{1}\left(t_{\max }\right)}, \quad \tau_{\mathrm{eff}}=12^{1 / 2} \beta_{2} t_{\mathrm{E}}, \\
t_{\max }=-b(\cos \theta) t_{\mathrm{E}} .
\end{gathered}
$$

\subsection{Finite Source Size Effects and Binary Rotation}

The analysis of $\S 2.1$ implicitly assumed point sources. The point-source approximation breaks down, however, when $u$ is $O(\rho)$, where $\rho$ is the radius of the source projected onto the lens plane in units of $r_{\mathrm{E}}$. In particular, for $u \lesssim \rho$, the magnification of a finite source differs substantially from that of a point source (Gould 1994; Nemiroff \& Wickramasinghe 1994; Witt \& Mao 1994; Witt 1995). Since, for a fixed perturbation size $\delta_{\max }$, a smaller flux ratio requires that the secondary approach closer to the lens, there will be a lower limit on $\epsilon$ below which equations (2.5) are no longer valid.

Given the small flux ratios involved, the secondary source will likely be a main-sequence star of solar luminosity or less. Thus I adopt a source radius of $R_{\odot}$, which at a distance of $8 \mathrm{kpc}$, for a typical bulge self-lensing event with $t_{\mathrm{E}} \sim 20$ days, $v \sim 200 \mathrm{~km} \mathrm{~s}^{-1}$, and $D_{\mathrm{ol}} \sim 6 \mathrm{kpc}$, translates to $\rho \sim 10^{-3}$. Thus equations (2.5) are not valid for those events with $\beta_{2} \lesssim 10^{-3}$. In order to produce perturbations with $\delta_{\max }>0.05$, the secondary must have an impact parameter $\beta_{2} \lesssim 20 \epsilon$. Thus equations (2.5) are not valid for binary sources with $\epsilon_{2} \lesssim 10^{-4}$. For flux ratios larger than this, finite source effects can be safely disregarded, and equations (2.5) are valid.

The effects of the rotation of the binary source for perturbations of this type can be entirely disregarded. The justification for this is as follows. To first order, the curvature of the path of the secondary due to rotation during the perturbation can be ignored. Thus the only effect is that the transverse velocity is now given by $v=\left|v_{0}+v_{2}\right|$, where $v_{0}$ is now the transverse velocity of the primary and $v_{2}$ is the velocity of the secondary relative to the primary. The timescale of the perturbations will be changed, since $\tau_{\text {eff }}=12^{1 / 2} \beta_{2} r_{\mathrm{E}} / v$. However, this effect can be reproduced by simply changing the value of $\beta_{2}$. The observed value of $\delta_{\max }$ can then be reproduced by changing $\epsilon$. Therefore a perturbation with observables $\tau_{\text {eff }}$ and $\delta_{\text {max }}$ can be produced by a static binary with parameters given by equation (2.5), or by a rotating binary with slightly different values of $\epsilon$ and $\beta_{2}$. Thus, to first order, the effect of rotation is entirely unobservable. The second-order effect is the curvature of the path of the secondary during the perturbation, which will produce effects that cannot be reproduced by parameter variations as they can for the first-order effect. This curvature is given by the square of the amount the binary source rotates during the course of the perturbation, $\psi^{2}=\left(2 \pi \tau_{\text {eff }} / P\right)^{2} \simeq$ $\left(22 \beta_{2} t_{\mathrm{E}} / P\right)^{2}$, where $P$ is the period of the binary source. Toward the Galactic bulge, the typical event timescale is 
$t_{\mathrm{E}} \sim 20$ days (Alcock et al. 1997a). For bulge self-lensing events, $v \sim 200 \mathrm{~km} \mathrm{~s}^{-1}$, and thus $r_{\mathrm{E}} \sim 2.3$ AU. Using Kepler's law, and assuming a binary source with separation $b=r_{\mathrm{E}}$ at $8 \mathrm{kpc}$ and total mass $M=2 M_{\odot}$, and a lens at 6 $\mathrm{kpc}$, I find a binary source separation projected into the source plane of $3 \mathrm{AU}$, and a period of $P \sim 3.7 \mathrm{yr}$. Thus $\psi^{2} \sim 0.1 \beta_{2}^{2}$. The perturbations considered here require $\beta_{2} \ll 1$, and thus the amount the binary source rotates during the perturbations is entirely negligible.

\section{PLANETARY MICROLENSING AND THE BASIC DEGENERACY}

Planetary microlensing events are a subset of binary microlensing events with small mass ratio of the binary, $q \ll 1$. These are characterized by small perturbations to the standard microlensing curve. The exact solution to the magnification of a binary lens has no analytic form, and is therefore difficult to study systematically. However, as Gould \& Loeb (1992) and Dominik (1996) have shown, when $q \rightarrow 0$, the binary lens reduces to a Chang-Refsdal lens (single lens plus external shear; see Chang \& Refsdal 1979). Thus for $q \ll 1$, a binary lens can be approximated by a Change-Refsdal lens. With this approximation, it is possible to obtain analytic expression for two of the quantities of interest, $\tau_{\text {eff }}$ and $t_{\max }$. This vastly simplifies the comparison between the binary-source perturbations discussed here and planetary events. For $q \simeq 10^{-3}$ and most binary separations, this is a fair approximation. Furthermore, the approximation improves as $q$ decreases, and is exactly in the limit $q \rightarrow 0$ (Dominik 1998). Since I am only concerned with obtaining approximate relations between binary-source and planetary perturbations, and in gaining some insight into the nature of the degeneracy between them, I will use this approximation throughout.

As with binary-source perturbations, the gross features of planetary lens perturbations can be described by three parameters: the maximum deviation, $\delta_{\max }$; the FWHM; and the time of maximum deviation, $t_{\max }$. In general, $\delta_{\max }$ is a function of the geometry of the event; the FWHM is given roughly by $\tau_{\text {eff }} \sim q^{1 / 2} t_{\mathrm{E}}$, where $t_{\mathrm{E}}$ is the timescale of the main light curve; $t_{\max }$ is a function of the planet-star projected separation $y$ in units of the Einstein ring, and the geometry of the event, $t_{\max } \simeq y^{-1}\left(y^{2}-1\right) \cos (\phi) t_{\mathrm{E}}$, where $\phi$ is the angle between the planet-star axis and the direction of source motion. Thus, a planetary event is described by (Gaudi \& Gaudi 1997b)

$$
\tau_{\text {eff }} \sim q^{1 / 2} t_{\mathrm{E}}, \quad t_{\max } \simeq y^{-1}\left(y^{2}-1\right) \cos (\phi) t_{\mathrm{E}},
$$

along with $\delta_{\max }$, which specifies the exact geometry. To be precise, the expression for $t_{\max }$ in equation (3.1) is the time when the trajectory exactly crosses the center of the planetary caustic in the Chang-Refsdal limit. A better approximation can be obtained by replacing $y$ in equation (3.1) by $y+\alpha$, where $\alpha$ is specified by $\delta_{\max }$. However, for $\delta_{\max } \gtrsim 0.05$, $\alpha \lesssim 0.1$, and hence I will ignore it here.

Here I have ignored finite-source effects. For $q \lesssim 10^{-4}$ (Neptune mass or smaller), finite-source effects become significant; however, as I discuss in $\S 5.1$, the severity of the degeneracy is reduced when finite-source effects are taken into consideration. Thus, for Jupiter-mass planetary perturbations, the following analysis is entirely applicable, whereas for perturbations arising from planets of Neptune mass or smaller, the analysis makes the degeneracy seem somewhat worse than it actually is.
Consider, for example, a perturbation with observables $\tau_{\text {eff }}=0.03 t_{\mathrm{E}}, \delta_{\max }=0.16$, and $t_{\max }=0.37 t_{\mathrm{E}}$, superimposed on a primary light curve with $\beta=0.37$. Then, from equation (3.1), a planetary event with $q \sim 10^{-3}, y \sim 1.3$, and $\phi \sim 45^{\circ}$ will reproduce the observed values of $\tau_{\text {eff }}, \delta_{\max }$, and $t_{\max }$. On the other hand, using equation (2.5), a binary-source event with $\epsilon \sim 5 \times 10^{-3}, b \sim 0.5$, and $\theta \sim-44^{\circ}$ would also reproduce the observables. Thus, at the level of the gross features $\left(\delta_{\max }, t_{\max }\right.$, and $\left.\tau_{\text {eff }}\right)$, the binary-source and planetary models will provide equally satisfactory fits to the observed perturbation. This is the basic degeneracy, and the example above is illustrated in Figure 1. Note that the maximum difference between the planetary and binarysource light curves is $\sim 4 \%$. Also shown in Figure 1 is the light curve obtained using the exact binary formalism, with binary parameters $q=10^{-3}, \theta=45$, and $b=1.12$ (the discrepancy in the value of $b$ is due to the term $\alpha$ discussed above). Obviously the difference between the light curves obtained using the Chang-Refsdal approximation and the
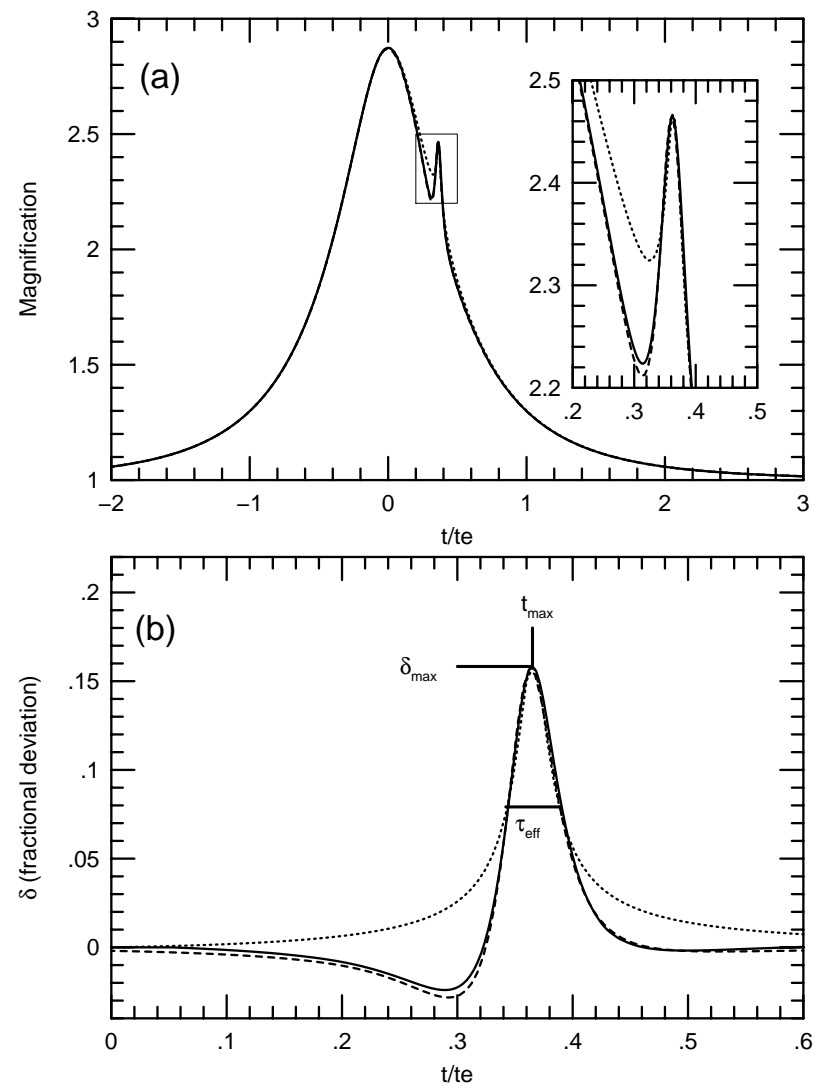

Fig. 1.-(a) Magnification as a function of time in units of the Einstein ring crossing time, $t_{\mathrm{E}}$, for a planet/star system (solid curve) with a mass ratio $q=10^{-3}$, a separation in units of the Einstein ring of $y=1.3$, and angle between the planet-star axis and direction of source motion $\phi=45^{\circ}$, and for a binary-source system (short-dashed curve) with flux ratio $\epsilon=5 \times 10^{-3}$, projected separation in units of the Einstein ring $b=0.5$, and angle between the binary-source axis and the direction of source motion $\theta=44^{\circ}$. The inset shows a detail of the light curves around the time of the perturbation. Also shown is the magnification for the planet/ star system calculated using the full binary formalism (long-dashed curve). (b) Fractional deviation from the main point-mass point-lens light curve as a function of time in units of $t_{\mathrm{E}}$ for the two light curves in $(a)$. Both planetary (solid curve) and binary-source (short-dashed curve) perturbations have the same observables $\tau_{\text {eff }}=0.03 t_{\mathrm{E}}$, the full-width half-maximum of the perturbation, $\delta_{\max }=0.16$, the maximum fractional deviation, and $t_{\max }=0.37 t_{\mathrm{E}}$, the time of maximum deviation. Also shown is the fractional deviation for the planet/star system calculated using the full binary formalism (long-dashed curve). 
full binary formalism is negligible in comparison to the effect discussed here.

From the example above and the discussion in $\S 2$, it is apparent that the basic requirements for a binary-source light curve to mimic that of a planetary event are a small flux ratio $\epsilon$ and a specific geometry, i.e., one in which the fainter source passes very close to the lens. More specifically, from equations (2.5), the binary-source parameters required to reproduce an event with observables $\tau_{\text {eff }}, \delta_{\max }$, and $t_{\max }$ are

$$
\begin{gathered}
\epsilon=\frac{\tau_{\mathrm{eff}}}{t_{\mathrm{E}}} \frac{\delta_{\max } A_{1}\left[u_{1}\left(t_{\mathrm{max}}\right)\right]}{12^{1 / 2}}, \quad b=\frac{t_{\max }}{t_{\mathrm{E}} \cos \theta}, \\
\theta=\tan ^{-1}\left(\frac{-\beta_{1} t_{\mathrm{E}}}{t_{\max }}\right),
\end{gathered}
$$

where, as before, $A_{1}$ is given by equation (2.1) evaluated at $t_{\max }$, and where now $t_{0}=0$. It is apparent that the value of $b$ required to fit an observed perturbation is fixed by the geometry through the observables $\beta_{1}$ and $t_{\max }$. The required value of $\epsilon$, however, depends not only on the geometry, but also on the observed $\delta_{\max }$ and $\tau_{\text {eff }}$. Furthermore, since the geometry of the event affects $\epsilon$ only through $u_{1}\left(t_{\max }\right)$, and $u_{1}\left(t_{\max }\right)^{2}=\left(t_{\max } / t_{\mathrm{E}}\right)^{2}+\beta_{1}^{2}$, changing $t_{\max } / t_{\mathrm{E}}$ has the same effect on $\epsilon$ as changing $\beta_{1}$.

Figure 2 shows contours of the difference in magnitude between the two sources, $\Delta V=-2.5 \log \epsilon$, required to reproduce the given $\tau_{\text {eff }}$ and $\delta_{\max }$, for three different geometries: (1) $\beta_{1}=0.3, t_{\max }=0.3 t_{\mathrm{E}}$; (2) $\beta_{1}$ or $t_{\max } / t_{\mathrm{E}}$ smaller by 0.05 ; (3) $\beta_{1}$ or $t_{\mathrm{max}} / t_{\mathrm{E}}$ larger by 0.05 . A large range of magnitude differences, $\Delta V \sim 9-5$, can produce perturbations with $\delta_{\max }$ and $\tau_{\text {eff }}$ in the ranges produced by planetary microlensing events. For clump giant primaries (spectral type $\mathrm{K}$

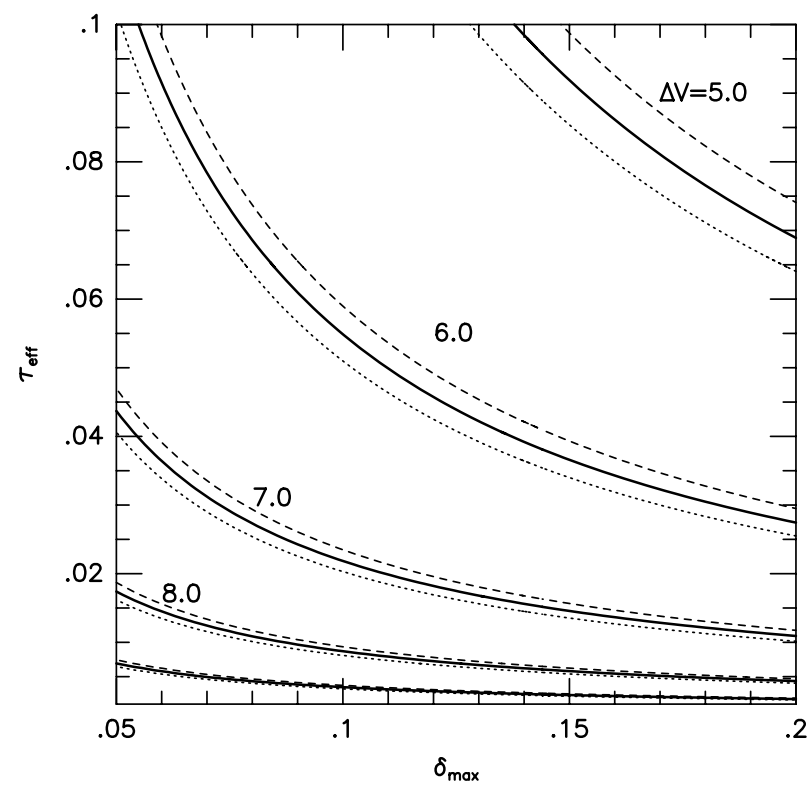

FIG. 2.-Contours of the difference in magnitude between the two sources, $\Delta V$, required to produce perturbations with the given full-width half-maximum, $\tau_{\text {eff }}$, and maximum fractional deviation, $\delta_{\max }$. The contours have spacings of $1 \mathrm{mag}$. The solid contours are for the geometry where the primary source has a impact parameter $\beta_{1}=0.3$ and the time of maximum fractional deviation in units of the Einstein ring crossing time is $t_{\max } / t_{\mathrm{E}}=$ 0.3 . The dotted contours are for the geometry where either $\beta_{1}$ or $t_{\max } / t_{\mathrm{E}}$ is smaller by 0.05 , and the dashed contours are for the geometry where either $\beta_{1}$ or $t_{\mathrm{max}} / t_{\mathrm{E}}$ is larger by 0.05 .
III, $M_{V} \sim 1$ ), this range in $\Delta V$ corresponds to secondaries of spectral type anywhere from solar $(\mathrm{G} \mathrm{V})$ to late dwarfs (M V).

\section{EXTREME FLUX RATIO BINARY-SOURCE EVENT PROBABILITIES}

For a binary source with $\epsilon \ll 1$ to be detected, the lens must pass close to the secondary. The probability that a trajectory with any $\beta_{1} \leq 1$ will pass within $\beta_{2}$ of the secondary is $\sim \beta_{2}$. Consider a binary source with $\Delta V=5$. The secondary must have $\beta_{2} \lesssim 0.1$ to produce perturbations with $\delta_{\max } \gtrsim 0.05$. Thus the detection probability for a binary source with $\Delta V=5$ is $\sim 20 \%$. A more careful treatment must take into account the fact that the magnitude of the perturbation depends on the time of the perturbation relative to the primary light curve (see equations [2.5]). This effect will serve to reduce the detection probability relative to the naive estimate. To quantify this, I calculate, for a given $\epsilon$ and $b$, the fraction of binary-source events that lead to detectable perturbations. Although planetary events can produce a wide range of maximum deviations, events with $\delta_{\max }<5 \%$ are unlikely to be detected. I therefore assume that the event is detected if $\delta_{\max }>0.05$. I place the additional constraint that $t_{\max } / t_{\mathrm{E}} \geq-1$, since perturbations are unlikely to be detected before the main event begins. To calculate the fraction, I integrate over $0 \leq \theta<2 \pi$ and $0 \leq$ $\beta_{1} \leq 1.0$. The detection probability is simply the number of events that satisfy the detection criteria divided by the total number of trial events. Figure 3 shows the fraction of events that lead to perturbations with parameters given above, for $\Delta V=4-9$, and $b=0-3.0$. For $\Delta V=4$, the detection probability can be quite high, $\sim 30 \%$. Even for $\Delta V=7$, the probability is nonnegligible and is a few percent.

A number of authors have calculated the detection probability for planets based on similar detection criteria. Gould \& Loeb (1992) found that, for Jupiter-mass planets with

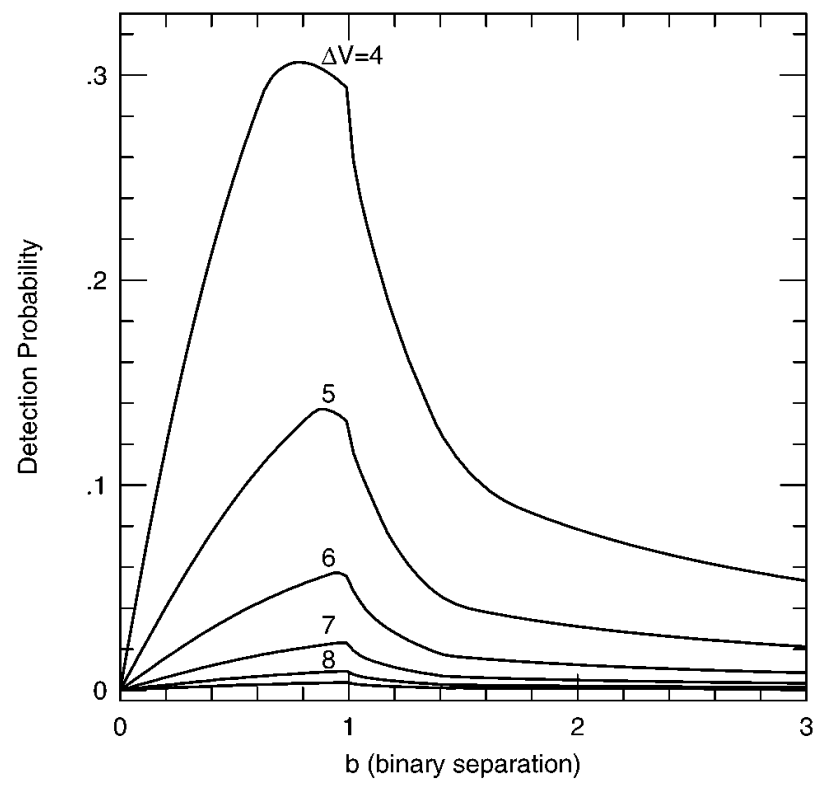

FIG. 3.-Fraction of binary-source events that will be detected for the given values of the difference in magnitude between the sources, $\Delta V$, as a function of the projected separation $b$ of the sources in units of the Einstein ring, for $\Delta V=4-9$. A binary source is considered detected when the perturbation meets the detection criteria for the maximum fractional deviation, $\delta_{\max } \geq 0.05$, and the time of maximum deviation, $t_{\max } / t_{\mathrm{E}} \geq-1$. 
projected separations $0.5 \lesssim y \lesssim 1.5$, the probability is $\sim 15 \%-20 \%$. For Earth-mass planets with $0.5 \lesssim y \lesssim 1.5$, Bennett \& Rhie (1996) found detection probabilities of $\sim 1 \%-3 \%$. Since these detection probabilities are of the same order of magnitude as the detection probabilities for binary-source perturbations with $\Delta V=4-7$ and $0.5 \gtrsim b \gtrsim$ 1.5 , if binary sources with these flux ratios and projected separations are at least as ubiquitous as the planets the monitoring campaigns hope to detect, they will provide a serious contaminating background.

\section{BREAKING THE DEGENERACY}

As shown, in $\S 4$, it is likely that binary sources will provide a significant contaminant in a sample of suspected planetary events. It is therefore essential that efforts be made to resolve this degeneracy. Here I discuss three possible methods to do this: detailed light curves, color information, and spectroscopic information. Each method has significant limitations. It is therefore essential that, whenever possible, all the following methods be used to ensure that this degeneracy is broken and that any planet detection is secure.

\subsection{Detailed Light Curves}

The most obvious way to break the degeneracy is to obtain accurate and dense sampling of the light curve. As is apparent from Figure 1, although a binary source and a planetary lens can produce perturbations with the same basic features $\left(\tau_{\mathrm{eff}}, \delta_{\max }\right.$, and $\left.t_{\mathrm{max}}\right)$, the detailed light curves are dissimilar. In particular, during the wings of the perturbation, a planetary event often produces negative deviations of a few percent, whereas binary-source perturbations produce only positive perturbations. For planets of $q \lesssim 10^{-4}$, finite source effects serve to increase the magnitude of the negative deviations during the wings of the perturbation, thereby making the binary-source and planetary perturbation more dissimilar. Thus, if one could resolve the observed light curve to better than the $\sim 4 \%$ level during the wings of the perturbation, the degeneracy would be broken. One would require dense and regular sampling of the curve, however, since the two cases are significantly $(>4 \%)$ different only during the first wing, and then only for a short time $\left(\sim 0.1 t_{\mathrm{E}}\right.$, or $\sim 1$ day for typical parameters).

In fact, there exist two types of planetary perturbations: those which perturb the major image of the source formed by the primary lens, and those which perturb the minor image. Minor image perturbations are characterized by large $(5 \%-20 \%)$ negative deviations. Binary-source perturbations are therefore incompatible with minor image planetary perturbations, and there exists no degeneracy. Unfortunately, minor image perturbations are likely to represent considerably less than half of all detected planetary perturbations, since minor image perturbations have a much smaller intrinsic detection probability for large mass ratio planetary systems (Gould \& Loeb 1992). For smaller mass ratios, finite source effects will decrease the fraction of minor image perturbations even further (Bennett \& Rhie 1996).

\subsection{Color Information}

A second way to break the degeneracy is to use color information. If the perturbation is due to a binary source, and the sources have different colors, there will be a color change during the course of the perturbation. Suppose that the binary source has an (unlensed) magnitude difference $\Delta V=\left(V_{2}-V_{1}\right)$ in $V$-band and $\Delta H=\left(H_{2}-H_{1}\right)$ in $H$-band. Then I define $\epsilon_{V}=10^{-0.4 \Delta V}$ and $\epsilon_{H}=10^{-0.4 \Delta H}$. The color change during the event is, $\Delta(V-H)=2.5$ $\log \left(A_{\mathrm{tot}, H} / A_{\mathrm{tot}, V}\right)$, where $A_{\mathrm{tot}, V}$ and $A_{\mathrm{tot}, H}$ are given by equation (2.3), with the appropriate $\epsilon$. Using the relation $\delta \simeq\left(A_{\text {tot }}-A_{1}\right) / A_{1}$, this becomes

$$
\Delta(V-H) \simeq 2.5 \log \frac{\delta_{V}+1}{\delta_{H}+1} .
$$

Using the relation for $\delta$ from equation (2.5), and defining $r \equiv \epsilon_{H} / \epsilon_{V}$, I rewrite this for the two cases $r<1$ and $r>1$ :

$$
\Delta(V-H)= \begin{cases}2.5 \log \frac{\delta_{V}+1}{r \delta_{V}+1}, & r<1, \\ 2.5 \log \frac{\delta_{H} / r+1}{\delta_{H+1}}, & r>1 .\end{cases}
$$

Note that $2.5 \log r=(V-H)_{2}-(V-H)_{1}$, i.e., the ratio $r$, is simply related to the color difference between the secondary and the primary. The maximum color change occurs at the peak of the perturbation and can be found by replacing $\delta_{V}$ in equation (5.2) by $\delta_{\max , V}$. In particular, note that for $r \ll 1$, $\Delta(V-H) \simeq 2.5(\ln 10) \delta_{V} \sim \delta_{V}$. Similarly, when $r \gg 1, \Delta(V$ $-H) \sim-\delta_{H}$. Thus the largest possible color change (in magnitudes) is equal to the maximum ( $V$ - or $H$-band) fractional perturbation.

Figure 4 shows the contours of $\Delta(V-H)$ for $\delta_{\max }=$ $0.05-0.20$ and $(V-H)_{2}-(V-H)_{1}=-2$ to 2 . For $\sim 1 \mathrm{mag}$ differences in the unlensed source colors, color changes of $\gtrsim 0.05 \mathrm{mag}$ are produced for all measurable perturbations. Even if the difference in source color is only $\sim 0.2 \mathrm{mag}$,

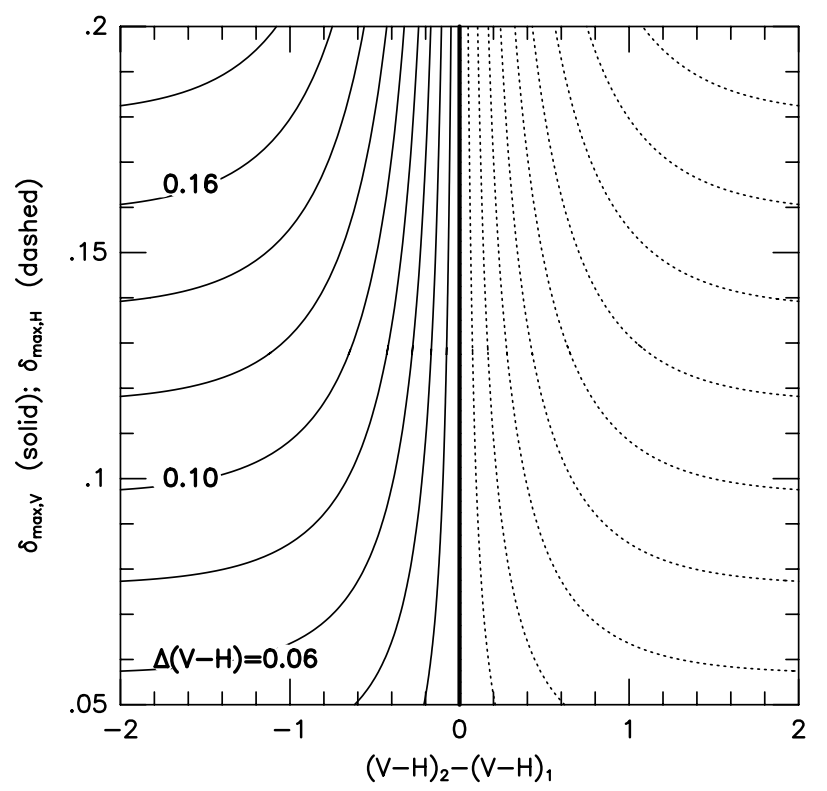

FIG. 4.-Contours of the maximum color shift $\Delta(V-H)$ in a binarysource event, as a function of the difference in colors of the two sources, $(V-H)_{2}-(V-H)_{1}$ and the size of the maximum fractional deviation, $\delta_{\max }$. The solid contours are for a shift to the blue, $\Delta(V-H)>0$, and dotted contours are for a shift to the red, $\Delta(V-H)<0$. If the secondary is redder than the primary, $(V-H)_{2}>(V-H)_{1}$, then $\Delta(V-H)<0$, and the maximum deviation will be in the $H$-band. Similarly, if the secondary is bluer than the primary, then the maximum deviation will be in the $V$-band. 
substantial ( $\gtrsim 0.05)$ color differences are produced for perturbations with $\delta_{\max } \gtrsim 0.1$. For perspective, I note that for a clump giant primary (K0 III, $M_{V} \sim 1, V-H \sim 2$ ), with a solar-type secondary (G V, $\left.M_{V} \sim 5, V-H \sim 1\right)$, the unlensed color difference is $\sim 1$ mag. For most binarysource pairs, therefore, a significant color shift will occur during the perturbation.

A color shift also occurs for planetary events with a small mass ratio. The form of this shift differs significantly from that of a binary source. At the beginning of the planetary perturbation, the color first shifts to the red; during the peak, it shifts to the blue; at the end of the perturbation, it shifts again to the red (see, e.g., Fig. 9 of Gaudi \& Gould 1997b). This is in contrast to binary-source perturbations, where the shift is always to either the red or the blue. Thus, a color shift for a binary source can be easily distinguished from that of a planetary event, and a measurement of a color shift during a perturbation would allow one to unambiguously distinguish between the two cases, and therefore break the degeneracy.

For planetary events with a large mass ratio, only a very small color shift is produced. Only a small color shift is produced for a binary source in which both sources have very similar colors. Thus if no color shift is detected it may appear that the degeneracy remains. In fact, this is not necessarily true, as there is likely to exist a correlation between the flux ratio and the color shift. Assume, for example, that the primary is known to be a $\mathrm{K}$ giant. Then, if the event is due to a binary source, the secondary is likely to be a mainsequence star. The color-magnitude relationship for mainsequence stars translates into a relationship between $\epsilon_{V}$ and $r$. This relationship, along with the value of $\epsilon_{V}$ measured from the observed light curve, allows one to estimate the expected color shift. If the observed color shift is inconsistent with this estimate, then the observed perturbation cannot be due to a binary source and the degeneracy is broken. There are situations, however, where even this argument would fail. For example, if the measured value of $r$ were consistent with the secondary being a $\mathrm{K}$ dwarf, one would expect no color shift, and therefore both binarysource and planetary origins of the perturbation would be consistent with the data, and the degeneracy would remain. In this situation, one would be forced to rely on either the detailed light-curve information, or, as described in the next section, spectroscopic information.

\subsection{Spectroscopic Methods}

If the methods suggested in $\S \S 5.1$ and 5.2 fail, there remain other methods to break the degeneracy. One possible method is to take spectra of the source both during and after the perturbation. If the perturbation is due to a binary source, both sources will be contributing to the spectrum during the perturbation, whereas after the perturbation, only the primary will contribute significantly to the spectrum. Thus, if the binary source is a giant-dwarf pair (as it is likely to be), then the equivalent widths of pressuresensitive spectral features will differ between the two spectra.

Finally, one could monitor the source both photometrically and spectroscopically after the event and search for any signs of binarity induced by orbital motion and/or eclipses. Given that there will likely be only a handful of candidate planetary perturbations, this should not require a substantial amount of telescope resources.

\section{PROPER MOTIONS}

If it is determined that an observed perturbation is due to a binary source rather than a planet, one can derive additional information about the lens. From the observed light curve, of a binary-source event, one can obtain the observables $t_{\mathrm{E}}, \beta_{1}, \beta_{2}, t_{0}$ and $t_{\max }$. These observables are related to the physical projected separation, $l$, by (Han \& Gould 1997):

$$
l=\hat{r}_{\mathrm{E} \pm}\left[\left(\frac{t_{0}-t_{\mathrm{max}}}{t_{\mathrm{E}}}\right)^{2}+\left(\beta_{1} \pm \beta_{2}\right)^{2}\right],
$$

where $\hat{r}_{\mathrm{E}}=r_{\mathrm{E}}\left(D_{\mathrm{os}} / D_{\mathrm{ol}}\right)$ is the Einstein radius projected onto the source plane. If $l$ can be mesured by follow-up spectroscopy, then $\hat{r}_{\mathrm{E}}$ can be determined. As equation (6.1) stands, however, there exists a twofold degeneracy in the determination of $\hat{r}_{\mathrm{E}}$ due to the ambiguity in the impact parameter difference $\Delta \beta_{ \pm}=\left|\beta_{1} \pm \beta_{2}\right|$. However, for the binary-source events considered here, $\beta_{1} \gg \beta_{2}$, and thus $\Delta \beta_{+} \simeq \Delta \beta_{-} \simeq \beta_{1}$ and there exists no degeneracy.

I now discuss further the issue of determining $l$ from follow-up spectroscopy. In order to determine $l$, the orbital elements (intrinsic physical separation, eccentricity, true anomaly, etc.) must be determined, as must the inclination angle $i$ (see Han \& Gould 1997). The orbital elements can be determined from a complete radial velocity curve. After the microlensing event, only the spectral lines of the primary will be visible. For a circular orbit, the maximum velocity shift of these lines is

$$
\begin{aligned}
& v_{\max }=30 \mathrm{~km} \mathrm{~s}^{-1}(\sin i) b^{-1 / 2} \\
& \times\left(\frac{\mathscr{Q}_{M}}{\mathscr{Q}_{M}+1}\right)^{-1 / 2}\left(\frac{\hat{r}_{\mathrm{E}}}{\mathrm{AU}}\right)^{-1 / 2}\left(\frac{M_{1}}{M_{\odot}}\right)^{1 / 2} .
\end{aligned}
$$

Here $\mathscr{Q}_{M}=M_{1} / M_{2}$, and $M_{1}$ and $M_{2}$ are the masses of the primary and secondary, respectively. For a $\mathrm{K}$ giant primary with a solar-type secondary, $M_{1} \sim M_{\odot}$ and $\mathscr{Q}_{M} \sim 1$. For typical bulge self-lensing events, $\hat{r}_{\mathrm{E}} \sim 3$ AU. From Figure 3, the binary-source detection rate peaks at $b \sim 1$. Thus, for typical binary-source events of this type, the expected maximum velocity shift is $v_{\max } \simeq 12 \mathrm{~km} \mathrm{~s}^{-1}$ sin $i$. The period of such a system is $P \simeq 3.7$ yr. Excepting nearly face-on orbits, measurement of a complete radial velocity curve for such a system, while not trivial, is within current capabilities. The masses of the sources are known approximately from their luminosities and colors (see $\S 5.2$ ). The masses can be further constrained if a spectrum is taken at the time of the perturbation, since the lines of both sources will be apparent and the radial velocities of these lines give a direct measurement of the mass ratio $\mathscr{2}_{M}$. These masses along with the orbital elements determined from the observed radial velocity curve determine $i$ and thus yield a complete solution and a measurement of $l$. This, combined with the event observables $t_{\mathrm{E}}, \beta_{1}, \beta_{2}, t_{0}$, and $t_{\max }$, yield a measurement of $\hat{r}_{\mathrm{E}}$ via equation (6.1).

The fraction of events for which it is possible to measure $\hat{r}_{\mathrm{E}}$ by this method is likely to be small, $O(1 \%)$. I estimate this as follows. From Figure 3, the average detection rate for binary sources with $8 \lesssim \Delta V \lesssim 4$ and $0.5 \lesssim b \lesssim 1.5$ is $\sim 15 \%$. In a study of the multiplicity of $\mathrm{F}$ and $\mathrm{G}$ stars in the solar neighborhood, Duquennoy \& Mayor (1991) found that $\sim 40 \%$ of these stars had companions with masses from 0.1 to 1.1 times the mass of the primary. These types of systems will evolve into the giant/dwarf binaries relevant 
here. Of these multiple systems, they find that $\sim 10 \%$ have separations in the range where the binary-source detection probability is high, $0.5 \lesssim b \lesssim 1.5$. Thus I estimate that $\sim 0.15 \times 0.4 \times 0.1 \sim 1 \%$ of events should display binarysource perturbations that can be used to measure $\hat{r}_{\mathrm{E}}$.

The determination of $\hat{r}_{\mathrm{E}}$, along with parallax information gathered from either the Earth's motion (Gould 1992; Alcock et al. 1995; Buchalter \& Kamionkowski 1997) or from a parallax satellite (Refsdal 1966; Gould 1995;
Boutreux \& Gould 1996; Gaudi \& Gould 1997a), yields a complete solution of the lens parameters: mass, distance, and velocity (Gould 1996).

I would like to thank David Weinberg, Penny Sackett, and Darren Depoy for several stimulating discussions, and Andrew Gould for his careful reading of the manuscript. This work was supported in part by grant AST 94-20746 from the NSF.

\section{REFERENCES}

Alard, C. 1996, in IAU Symp. 173, Astrophysical Applications of Gravitational Lensing, ed. C. S. Kochanek \& J. N. Hewitt (Dordrecht: Kluwer), 214

Albrow, M., et al. 1996, in IAU Symp. 173, Astrophysical Applications of Gravitational Lensing, ed. C. S. Kochanek \& J. N. Hewitt (Dordrecht: Kluwer), 227

Albrow, M., et al. 1998, ApJ, submitted

Alcock, C., et al. 1995, ApJ, 454, 125 .1997a, ApJ, 479, 119 .1997b, ApJ, 491, 436

Ansari, R., et al. 1996, A\&A, 314, 94

Bennett, D., \& Rhie, H. 1996, ApJ, 472, 660

Bolatto, A., \& Falco, E. 1994, ApJ, 436, 112

Boutreux, T., \& Gould, A. 1996, ApJ, 462, 705

Buchalter, A., \& Kamionkowski, M. 1997, ApJ, 482, 782

Chang, K., \& Refsdal, S. 1979, Nature, 282, 561

Dominik, M. 1996, Ph.D. thesis, Univ. Dortmund .1997, A\&A, submitted

Duquennoy, A., \& Mayor, M. 1991, A\&A, 248, 485

Gaudi, B., \& Gould, A. 1997a, ApJ, 477, 152

. 1997b, ApJ, 486, 687

Gould, A. 1992, ApJ, 392, 442 ApJ, 421, L71

ApJ 441, L21 PASP, 108,465

Gould, A., \& Loeb, A. 1992, ApJ, 396, 104

Griest, K., \& Hu, W. 1993, ApJ, 397, 362

Han, C., \& Gould, A. 1997, ApJ, 480, 196

Mao, S., \& Paczyński, B. 1991, ApJ, 374, 37

Nemiroff, R. J., \& Wickramasainghe, W. A. D. T. 1994, ApJ, 424, L21

Peale, S. J. 1997, Icarus, 127, 269

Refsdal, S. 1966, MNRAS, 134, 315

Udalski, A., et al. 1997, Acta Astron., 47, 169

Wambsganss, J. 1997, MNRAS, 384, 172

Witt, H. 1995, ApJ, 449, 42

Witt, H., \& Mao, S. 1994, ApJ, 430, 505 